\title{
Conocimiento del espacio, representación y movilidad en las personas ciegas
}

\author{
ESPERANZA OCHAITA \\ JUAN ANTONIO HUERTAS \\ Universidad Autónoma de Madrid
}

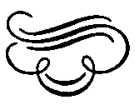

Resumen

\begin{abstract}
En este artículo se realiza una revisión exhaustiva y crítica de la literatura psicológica sobre el conocimiento del entomo en las personas invidentes. Se revisan los problemas que plantea la falta de visión en la actividad, representación y conocimiento de espacios cercanos (a los que el ciego accede con el tacto) y de entornos lejanos (en los que necesita movilidad), asi como las diferentes técnicas de entrenamiento en movilidad y conocimiento espacial. En las conclusiones se exponen los prablemas fundamentales que todavía hoy quedan sin aclarar y que, por consiguiente precisan de ulteriores investigaciones.
\end{abstract}

Palabras clave: Ceguera, conocimiento espacial, movilidad.

\section{Spatial knowledge, representation and mobility in blind people}

\section{Abstract}

In this article the psychological literature on the blind's environmental knowledge is critically reviewed. Topics such as the problems posed by lack of sight on the representation, knowledge and activity in close space (where the blind have to work tactually) and in distant environment (in which deambulation is necessary) are reviewed, as well as training technics for mobility and for improving the spacial knowledge of the blind. Finally, areas in which more research is needed are presented and discussed.

Key words: Blindness, spatial knowledge, mobility.

Agradecimientos: Este artículo constituye el inicio de un programa de investigación titulado «Ontogénesis y microgénesis de representación del entorno físico en las personas ciegas», financiado por la C.A.I.C.Y.T. del M.E.C. y dirigido por E. Ochaíta.

Dirección de los autores: Facultad de Psicología de la Universidad Autónoma de Madrid. Cantoblanco, 28049 Madrid.

Original recibido: Noviembre 1987. Revisión recibida: Febrero 1988. Aceptado: Marzo 1988. 


\section{INTRODUCCION}

Ha sido mucha la literatura que desde tiempos muy remotos se ha dedicado al problema de la espacialidad en las personas ciegas. Molineux, Locke, Berkely y Diderot ya trataron este tema y actualmente siguen apareciendo en las revistas psicológicas numerosos artículos dedicados a él. En la mayoría de los casos se ha tratado desde una perspectiva «visuocentrista» casi siempre basada en el prejuicio de que las imágenes son de origen visual y de que, en consecuencia, el conocimiento del espacio ha de ser prácticamente imposible en ausencia de visión. No obstante, si observáramos con cierto detenimiento las actividades que los invidentes realizan en su vida cotidiana, nos daríamos cuenta de que necesariamente han de tener un conocimiento espacial, ya que de ordinario reconocen formas y tamaños, realizan juicios de diferencias o semejanzas y caminan y se orientan en espacios más o menos grandes.

A pesar de la relevancia del tema, desde el punto de vista teórico, de la gran cantidad de trabajos que en la actualidad se siguen publicando al respecto (tal y como veremos en las páginas siguientes), y del punto de vista práctico, de las consecuencias que tiene para la educación de los invidentes, no contamos todavía con resultados concluyentes en temas tan importantes como pueden ser las relaciones entre el conocimiento del espacio cercano y el lejano, el papel de la experiencia visual en la realización de una amplia gama de tareas espaciales, los sistemas sensoriales implicados en la movilidad de los invidentes, la capacidad de estos para exteriorizar sus representaciones del ambiente, o el desarrollo del conocimiento del entorno en los niños privados de visión.

Así pues, con este artículo se pretende ofrecer un resumen de las investigaciones más importantes que relacionan de alguna manera espacio y ceguera, haciendo especial énfasis en aquellos aspectos que, como los citados en el párrafo anterior, precisan de investigaciones ulteriores. Se incidirá de forma especial en los temas que tengan o puedan tener mayor interés educativo para las personas invidentes.

\section{INVESTIGACIONES EN ESPACIO CERCANO}

La tarea de clasificar los numerosos y heterogéneos trabajos de investigación que se han realizado sobre la capacidad para comprender las distintas relaciones espaciales en ausencia de la visión resulta bastante compleja. Esto se debe, en gran medida, a que se parten de distintos presupuestos teóricos, se utilizan diseños heterogéneos e, incluso, diversas terminologías (Ochaita, 1982).

Para los objetivos de este trabajo resulta conveniente hacer una primera distinción entre lo que se denomina «espacio cercano» y aespacio lejano».

Los trabajos de espacio cercano que ocupan este apartado estudian problemas de reconocimiento y representación espacial en el área que las personas invidentes pueden abarcar con los brazos, tomando la información mediante el tacto activo. Las investigaciones sobre espacio lejano, a las que nos referiremos más tarde, analizan las relaciones espaciales de entornos más o menos grandes (un laboratorio, un colegio, un pueblo, etc.) que no son abarcables mediante los brazos, y que por tanto, sólo son accesibles para las personas carentes de visión utilizando información de otras modalidades sensoriales, como la audición, el movimiento y la propiocepción. 
Por tanto, para los ciegos las relaciones entre el espacio cercano y el lejano han de ser más complejas que para los videntes. Mientras que para estos últimos los dos tipos de relaciones espaciales implican un único sistema sensorial: la visión (aunque en el espacio lejano intervengan de modo subsidiario los otros sistemas sensoriales), para los invidentes el modo de acceder a ambos espacios implica la utilización de distintas fuentes de información (cinestésica, háptica, auditiva y propioceptiva). Volveremos no obstante a este problema en el apartado de conclusiones.

\section{Experiencia Visual y Rotación de Formas}

El sistema perceptivo háptico puede considerarse como un sentido espacial mediante el cual se pueden recoger aspectos tales como la forma y el tamaño de los objetos. Sin embargo muchos de los estudiosos del tema que nos ocupa (Worchel, 1951; Pick y Pick Klein, 1966, 1968; Marmor y Zaback, 1971; Jourmaa, 1973; O'Connor y Hermelin, 1975; Millar, 1976; Carpenter y Eisenberg, 1978) han dirigido sus investigaciones a intentar contestar a la siguiente pregunta: ¿Hasta qué punto es necesaria la experiencia visual para rotar mentalmente las formas en el espacio, tomando como referencia los ejes horizontal, vertical y oblicuo del espacio euclidiano?

A pesar de la complejidad del tema, podemos afirmar que los ciegos, incluso los que nunca han visto, son capaces de representarse mentalmente la rotación de las formas en el espacio, pudiendo tomar como referencia su propio esquema corporal, ya sea el eje horizontal/vertical que les proporciona la posición de todo el cuerpo o utilizando para orientarse una parte del mismo, como por ejemplo, el brazo o la mano (Carpenter y Eisenberg, 1978). El trabajo de estos autores es uno de los más completos que se han realizado sobre este tema. En el primer experimento presentaban a doce sujetos ciegos de edades entre los 15 y los 18 años dos letras en Braille, «P»y «F» en su imagen normal y en espejo en seis orientaciones cada una con un ángulo de 60 grados con respecto a la anterior. Los sujetos conocían la letra y únicamente debían de determinar si su imagen era o no la correcta. La medida de respuesta fue el tiempo de reacción. Los resultados mostraban que el tiempo de reacción aumentaba en función de la desviación respecto a la posición vertical hasta los 180 grados y después disminuía. De esto concluyen que la rotación mental puede tener lugar con una representación no visual. En el segundo y tercer experimento tratan de hacer una comparación cuantitativa con los sujetos videntes, los datos de las personas con uso de visión fueron muy semejantes a los de los ciegos, excepto en el caso de los videntes con los ojos tapados, que tardaron comparativamente más. En el cuarto experimento se trataba de averiguar si lo que se codifica como vertical en una tarea «háptica» depende de la posición de la mano, del cuerpo o de un marco de referencia externo como el suelo o la mesa. Para ello dividieron a los sujetos en dos grupos, el primero trabajaba con la mano en posición vertical (0 grados) y el otro a 300 grados, los resultados demostraron la influencia positiva de ese marco de referencia interno al sujeto.

Estos mismos autores mantienen además que existe una forma de representación háptica para tareas espaciales que no debe de ser ni relegada, ni olvidada. Incluso otros estudiosos mantienen que tal representación puede ser de un carácter más genérico, independientemente de la modalidad sensorial con que 
se tome la información, de naturaleza solamente espacial (Cooper y Shepard, 1973; Meltzlar y Shepard, 1974).

De todas formas no podemos pasar por alto el hecho de que la experiencia visual facilita la rotación mental, ya que los ciegos tardíos y los videntes a los que se les priva durante el experimento del uso de la visión, suelen tener menos problemas que los ciegos de nacimiento en la realización de este tipo de tareas. No obstante, no existe consenso sobre cuál es el período de tiempo mínimo con experiencia visual para que el sujeto tenga un desarrollo semejante al de los videntes (Marmor y Zaback, 1976; Millar, 1976; Schmitt y Warren, 1982; Dodds, 1983). La mejor realización en las personas con alguna experiencia visual puede verse matizada si consideramos que se ha demostrado en varias investigaciones (Jourmaa y Sounio, 1969; O'Connor y Hermelin, 1975; Millar, 1979, 1982) que se producen sobre todo ante formas visualmente familiares. Es decir, parece que en estos casos ocurre el fenómeno que se ha llamado «transposición» (Jourmaa, 1973; Hermelin y O'Connor, 1982), según el cual cuando la información proviene de un sistema perceptivo que no facilita el procesamiento de acuerdo con los requerimientos de la tarea, la información puede codificarse en términos de esquemas de conocimiento derivados de otra modalidad perceptiva más apropiada. Por contra, cuando a los sujetos con conocimiento visual se les presenta hápticamente objetos no conocidos visualmente, realizan la tarea de forma muy similar a los ciegos de nacimiento.

En estos casos las personas con conocimiento visual no pueden fácilmente transponer esos objetos a imágenes mentales. Por otro lado, estos datos los explican los autores por el uso de referentes visuales externos por parte de los sujetos con alguna experiencia visual (por ejemplo, la percepción óptica de las esquinas de una mesa), en contraposición con el uso de auto-referentes por parte de los ciegos congénitos (por ejemplo, la utilización del propio brazo). Pero la dicotomía entre referentes externos y auto-referentes es un tanto artificial, debido a que realmente en el espacio personal o cercano las relaciones espaciales no existen fuera del sujeto, esos referentes «externos» no tienen sentido sin el marco de referencia del propio sujeto (en nuestro ejemplo, la visión de las esquinas de la mesa varía en su orientación según la posición del propio cuerpo). Luego los sujetos ciegos de nacimiento pueden conocer mediante ciertas estrategias esos mismos referentes espaciales. El uso de las relaciones entre las claves propioceptivas, gravitacionales y el movimiento de las manos o brazos puede ser esa estrategia que les proporcione la retroalimentación que necesitan (Millar, 1982). Por tanto, y dado que no sabemos con certeza hasta qué punto esas diferencias entre ciegos congénitos y personas con alguna experiencia visual puedan deberse al aprendizaje, consideramos conveniente una educación que explote hasta el máximo esa modalidad de representación háptica a la vez que el conocimiento del esquema corporal en los niños ciegos.

No obstante, no hay que olvidar que el sistema perceptivo háptico tiene ciertas peculiaridades que lo diferencian del visual tanto en lo referente a la toma de información como a su procesamiento. Estas características que son fundamentalmente su secuencialidad, fragmentación y lentitud, pueden incidir en personas que, como los ciegos de nacimiento, no puedan trasponer la información táctil a imágenes visuales, imágenes que posteriormente guian la exploración. 
Desarrollo del Conocimiento Espacial

Otro tipo de estudios intentan inferir el conocimiento que el ciego tiene del espacio real, a partir de la realización de pruebas en que se presenta este mismo espacio en pequeña escala. En estos estudios (por ejemplo, Ochaita, 1982, 1984), basados generalmente en la teoría piagetiana de la evolución del conocimiento espacial, se presentan a los niños invidentes maquetas que representan problemas en los que están implicadas distintas relaciones espaciales. En general, puede afirmarse que los niños ciegos de nacimiento son capaces de comprender sobre los 11 años las relaciones espaciales topológicas (separación, proximidad, orden, cerramiento y continuidad), que son también las más sencillas para los niños videntes (más o menos sobre los 7 años). Las relaciones métricas o euclidianas (sistemas de referencias horizontal y vertical, paralelismos, ángulos, etc...), serían accesibles para los ciegos a partir de los 14 años, con un retraso respecto a los videntes de cinco a seis años. Pero lo importante en este tipo de problemas no está en el aspecto negativo que pudiera suponer tal retraso en los niños ciegos, sino el que sean capaces de llegar a comprender, sin entrenamiento, ese tipo de tareas. Es importante destacar el hecho de que los niños que nuncan han visto, llegan a comprender sobre los 14 a 15 años, problemas de perspectiva en pequeña escala, como el típico problema de «las tres montañas» de Piaget, utilizando relaciones espaciales no proyectivas, sino métricas o euclidianas.

Otros estudios (Simpkins y Siegel, 1979; Birns, 1986), basados también en la teoría piagetiana de la cognición espacial fundamental ${ }^{1}$, han obtenido resultados parecidos. No encontraron retrasos en la adquisición de las relaciones topológicas por parte de los ciegos cuando éstas se estudiaban utilizando la tarea de «percepción háptica», consistente en el reconocimiento háptico de diver: sas formas geométricas. Por el contrario hallaron dificultades en la mitad de los sujetos (de edades comprendidas entre los 6 y los 10 años) a la hora de realizar una tarea de espacio proyectivo. No obstante, estos autores ponen de manifiesto el hecho de que los invidentes pueden llegar a realizar pruebas de espacio proyectivo, aunque no explican en ningún momento las estrategias que utilizan para ello. Es también importante destacar que en la realización de tareas de espacio proyectivo Birns (1986) no encontrase diferencias entre ciegos de nacimiento y ciegos tardíos. En esta misma línea, Ochaita $(1982,1984)$ obtuvo resultados semejantes entre ciegos congénitos y los videntes que hacían la prueba con los ojos tapados. Esto puede sugerir que puede no ser la experiencia visual, sino el hecho de tomar la información con el tacto, lo determinante en la comprensión de este tipo de tareas espaciales en las que se hace difícil la transposición de los datos hápticos a imágenes visuales.

\section{INVESTIGACIONES EN ESPACIO LEJANO}

Pasemos ahora a analizar lo que se conoce como «espacio lejano», las relaciones espaciales en espacios grandes que no son abarcables mediante los brazos y que, en consecuencia, sólo son accesibles para las personas carentes de visión mediante la deambulación, partiendo de los datos que les proporciona el movimiento, la propiocepción y la audición. Los trabajos que abordan este tema son complejos de analizar ya que a menudo en ellos no se clarifica lo suficiente las diferencias entre actividad espacial, representación espacial y las formas de externalizar esas representaciones. 


\section{Orientación y Movilidad}

Uno de los aspectos que más importancia teórica y práctica tiene para la psicología de la ceguera, es el del desplazamiento y la orientación. La movilidad es una habilidad que requiere el funcionamiento de muy diferentes procesos, perceptivos, motores, asociativos, mnésicos, etc.; y por lo tanto de gran complejidad y de difícil estudio. Para los invidentes este proceso es especialmente complejo pues deben suplir con otros sentidos las importantes funciones que ejerce la visión. Todo movimiento está compuesto a la vez de aspectos espaciales y temporales, nos desplazamos siempre por un lugar, tardando más o menos tiempo en hacerlo. Hermelin y O'Connor (1975) y otros estudiosos han demostrado cómo ciertas características del estímulo se almacenan mejor si se recogen en una modalidad sensorial que en otra. Así las características espaciales del entorno se codifican mejor visualmente y las temporales de manera auditiva. Luego los ciegos, al carecer de codificación visual, tienden a utilizar lo auditivo-temporal, lo verbal como marco estructural más frecuente de representación de la deambulación.

Aún con el aprendizaje de algunas técnicas o estrategias en movilidad, los ciegos no consiguen un desplazamiento del todo adecuado, es decir, que sea tan independiente, seguro, confortable, y socialmente apropiado como el de los videntes (Foulke 1971, 1982; Suterko, 1973). Muchas veces esto ocurre porque el recorrido, la tarea que tienen que llevar a cabo, no les proporciona suficiente información como para que puedan codificarla adecuadamente; porque carecen de la experiencia, de los esquemas o de las estrategias adecuadas para que les permitan transponer esa información a otras modalidades más eficaces, como ocurre con las personas con experiencia visual. También estas deficiencias en movilidad pueden deberse a la ausencia de un entrenamiento adecuado. Por eso una gran mayoría de las investigaciones que se han llevado a cabo sobre el tema han tenido por finalidad única la evaluación de métodos de entrenamiento en movilidad, pero muchas veces lo han hecho con unos diseños experimentales inadecuados, y careciendo de una mínima apoyatura teórica, lo que ha originado que sus datos sean poco concluyentes.

Se puede considerar que en el proceso de la orientación y la movilidad en una ruta se encuentran implicados tres componentes (Rieser, Guth y Hill, 1982). En primer lugar, el caminante debe de conocer el trazado y las marcas o mojones que le permitan distinguir la ruta. En segundo lugar además el individuo debe ser capaz mientras camina de actualizar su posición en cada momento en relación con esos mojones. Los buenos viajeros, por último, usan al desplazarse de una amplia gama de conceptos generales y de sistemas conceptuales sobre el espacio (norte, sur, izquierda, a mano derecha, perpendicular a..., etc.). Teniendo en cuenta estos tres criterios, dentro del campo de la orientación y movilidad en invidentes se han establecido diferentes métodos para que los deficientes visuales obtengan y organicen los conocimientos sobre rutas y «mojones» adecuadamente. En cuanto a la actualización perceptiva han sido pocos los trabajos que lo han estudiado. En general, parece que este proceso pueden llevarlo a cabo los ciegos, aunque necesitan un poco más de tiempo, al tener que hacer elaboraciones cognitivas más complicadas que las puramente perceptuales, como es el caso de los videntes. Según nuestro criterio, hacen falta nuevas investigaciones sobre el problema de la actualización perceptiva que permitan conocer mejor esas estrategias que usan los invidentes. Para finalizar. y por lo 
que se refiere al tercer criterio, parece ser que aunque los ciegos utilizan y cono. cen algunos de esos conceptos espaciales por lo general no los usan con total efectividad.

Habida cuenta de las dificultades que tienen los ciegos para sus desplazamientos, han sido muchos los procedimientos y aparatos que se han creado para paliar de alguna forma esta deficiencia. Así hace ya tiempo que Richard Hoover diseñó, partiendo de sus observaciones en un hospital militar, un bastón más largo y más sensible al contacto con obstáculos, a la vez que creó un sistema de barrido que permitía una mayor seguridad en los desplazamientos de los invidentes. En las dos últimas décadas han ido apareciendo unos aparatos electrónicos que proporcionan alguna de la información espacial que se pierde por la carencia de la visión (Farmer, 1980; Weinstock, 1982; Coleman, Weinstock, 1984). Generalmente con estos aparatos se pretende, mediante ayudas acústicas, potenciar este sistema perceptivo en orden a permitir hacer estimaciones de distancias, de dirección, de posición, etc. Una de las funciones más importantes de estas ayudas electrónicas es la de proporcionar una mejor anticipación perceptiva. Esta anticipación hace posible el observar los objetos que van a ser necesarios para lina acción determinada antes de que se vaya a realizar. La anticipación perceptiva se lleva a cabo preferentemente con el sistema visual, por lo que las ayudas electrónicas les son útiles a los ciegos al permitirles detectar obstáculos y objetos que están a varios metros de ellos. Dentro de las ayudas electrónicas, tales como el Pathsounder, el Mowat Sensor, el Step Sensor,... quizá sea el Sonicquide el aparato más conocido y el más versátil -informa no sólo de la aparición de un obstáculo, sino que además es capaz de estimar distancias y posiciones de los objetos en el espacio, pudiendo proporcionar incluso alguna información acerca de ciertas características de las superficies-. Sin embargo, algunos autores como Foulke (1982) muestran ciertas reservas hacia ellas, primero porque no están del todo desarrolladas y perfeccionadas y sobre todo porque no han tenido muy en cuenta, ni han analizado qué información espacial es la crítica para una movilidad correcta, quizá por que todavía no existe una teoría del espacio lo suficientemente amplia y fuerte que permita establecer esas ideas.

\section{Representación Espacial y Mapas Cognitivos}

Vamos ahora a repasar los datos existentes acerca de la «representación del entornos. Se trata, por tanto, de saber qué tipo de representaciones tienen los invidentes de su medio ambiente más o menos inmediato, como la casa donde viven, el colegio, el barrio o la ciudad. Von Senden en 1960 concluía de todas sus observaciones que no es posible tener una verdadera representación del espacio en ausencia de visión. Anteriormente Rèvèsz (1950) había propuesto que sin visión podía existir una representación del espacio pero que ésta debía de estar empobrecida. En una investigación realizada por Kephart y sus colaboradores (1974) con niños invidentes de edades comprendidas entre los 5 y los 7 años, se encontró que la representación del entorno era más pobre en los niños ciegos que en los videntes de la misma edad. Tal pobreza se manifestaba en la descripción de las partes del cuerpo, de la estructura anterior de la casa, del barrio y de la calle. Pero como veremos más adelante los datos actuales no son tan decepcionantes para los sujetos privados de visión.

Tanto en los ciegos como en los videntes las representaciones que se adquie- 
ren al viajar muy probablemente sean esquemas que provienen de muy diferentes modalidades (Schmidt, 1976), esto es, resúmenes y abstracciones de la información que necesitan para desplazarse independientemente. Aunque la polémica sobre de qué forma se almacena en el individuo el conocimiento espacial (cfr. por ejemplo De Vega, 1984) no está del todo resuelta, algunos autores (Appleyard, 1970; Shagen, 1970; Evans, Marrero y Butler, 1981; Foulke, 1982; Millar, 1982) afirman que ésta es selectiva dependiendo de la información espacial que se almacene, unas veces se hará por medio de imágenes mentales y otra por medio de proposiciones. Es decir, los diferentes tipos de imágenes mentales son una forma opcional de codificar, que deriva indirectamente de los modos de experiencia, pero que depende de variables tales como la tarea y el propio conocimiento (Millar, 1982).

Cuando un caminante se desplaza por una ruta que no conoce se ayuda para llegar a su destino de la información que recibe del entorno durante su desplazamiento y de las generalizaciones o conocimientos espaciales que posee. Ios invidentes en este caso se desplazarán con menos seguridad porque recogen menos información del entorno y poseen una menor anticipación perceptiva. Además, debido a que en sus representaciones del entorno no se incluyen ciertos objetos que sirven de «mojones» o indicadores para los videntes, les es más difícil el mantener una orientación correcta (Foulke, 1982). Es más, el mismo autor hipotetiza que los invidentes no pasarán de tener una estructura de «rutas», primera etapa del aprendizaje de un espacio lejano, en contraposición con los videntes que reemplazan esta representación con otra más elaborada, en términos de mojones relacionados espacialmente. Por tanto los videntes dependen menos de sus representaciones del espacio para desplazarse, ya que suelen obtener del entorno la información que necesitan mientras que desarrollan la tarea. Los ciegos, por el contrario, dependen más de las representaciones, de los razonamientos inductivos que hagan de su percepción, que obligatoriamente es serial y fragmentaria (Hollyfield y Foulke, 1983). Pero lo dicho hasta aquí no significa que los ciegos sean incapaces de moverse en rutas desconocidas, las diferentes investigaciones que se han realizado han puesto de manifiesto que los ciegos, incluso los que lo son desde el nacimiento, son capaces de elaborar «mapas cognitivoss o «representaciones topográficas» de los ambientes en que se mueven (Casey, 1978; Rieser, Lockman y Pick, 1980; Dodds, Howarth y Carter, 1982; Hollyfiel y Foulke, 1983). Son capaces también de aprender la estructura de una nueva ruta casi con la misma rapidez que los videntes y pueden, basándose únicamente en el razonamiento, colocar ciertos mojones correctamente sin haberlos percibido (Hollyfield y Foulke, 1983). Sin embargo la experiencia visual puede facilitar la representación del entorno, ya que los invidentes que la han tenido suelen hacer exteriorizaciones más adecuadas de sus representaciones que los ciegos de nacimiento (Casey, 1978).

Otra de las variables que necesariamente han de incidir en la capacidad de los ciegos para representarse el entorno es el tamaño de ese espacio. Es muy posible que los invidentes tengan una representación adecuada de los espacios relativamente pequeños (por ejemplo, un barrio) y sin embargo tengan problemas para coordinar esos espacios entre sí (una ciudad). Dado que en las investigaciones anteriormente citadas se comparan espacios de muy distintos tamaños, esa puede ser una de las causas de la heterogeneidad de algunos resultados.

Por lo que hemos dicho los ciegos tienen menos conocimiento que los videntes acerca de la identidad y disposición de los objetos que configuran un espa- 
cio. Las guías acústicas a las que antes nos referimos les podían proporcionar la ayuda que necesitan para obtener información de ese tipo. Además la perfección en las representaciones parece estar en función de la actividad real, de la movilidad del invidente en el ambiente de que se trate, por lo que el entrenamiento puede jugar un papel muy importante en orden a mejorar esas representaciones.

La externalización de las representaciones espaciales de los sujetos, es decir, las distintas formas de objetivar sus representaciones del entorno, en el caso de los ciegos, por razones obvias, se hace más difícil que con los videntes. Distintos experimentos han conseguido adaptar técnicas de dibujo que fuesen útiles a los invidentes para representar una ruta (Dodds, Howarth y Carter, 1982). Otros autores han resuelto ese problema evaluando la precisión de esas representaciones por medio de estimaciones de distancias (Lockman, Rieser y Pick, 1981), o utilizando descripciones y cuestionarios verbales (Rosencranz y Suslick, 1976). Pero a las dificultades que la ausencia de visión genera en la externalización, hay que añadirle el problema metodológico que trae consigo el hecho de trabajar con esos tipos concretos de externalizaciones (Evans, 1980; Marchesi, 1983). En ese sentido, nos parecen más adecuadas otras técnicas que se han aplicado para plasmar esas representaciones. Así, Hollyfield y Foulke (1983) utilizaron maquetas magnéticas de fácil discriminación táctil y Rieser Lockman y Pick (1980) usaron para provocar la externalización estimaciones de distancias con escalas multidimensionales.

Otros estudios han intentado trasponer el conocimiento sobre las relaciones espaciales de unos objetos en un espacio cercano, un tablero, a un espacio real y amplio, una habitación. Los resultados que se han obtenido han sido contradictorios: mientras que en el estudio de Fletcher (1981) no se encontró ninguna utilidad a este procedimiento, en el trabajo de Herman, Herman y Chatman (1983) los sujetos fueron capaces de deducir todo el conjunto de relaciones en un medio a gran escala con bastante precisión. La razón de tal discrepancia la explicaban los autores en virtud del número de objetos y relaciones que se establecían en el modelo a pequeña escala. De lo que se puede deducir que los mapas tactiles pueden ser útiles para introducir a los deficientes visuales en las relaciones generales entre objetos (más que en las específicas) en medios a gran escala, siempre y cuando el número de relaciones y objetos no sea muy numeroso. Por otra parte, en la utilización de este tipo de mapas ha de tenerse en cuenta la edad de los sujetos. En diversos estudios realizados con niños videntes se ha puesto de manifiesto que sólo a partir de los 11 años empiezan los niños a ser capaces de comprender representaciones cartográficas convencionales y los subsecuentes problemas de escala, localización y dirección que estos implican (Ochaita, 1986).

Así pues, hay que tener en cuenta, además, que la capacidad para representarse el entorno evoluciona con la edad (Appleyard, 1970; Siegel y White, 1975; Evans, 1980). En primer lugar los niños pequeños perciben y recuerdan los mojones. Luego son capaces de aprender una ruta. Después empiezan a coordinar, sin una buena organización interna, los mojones y las rutas. Finalmente la representación se consigue cuando las rutas se coordinan dentro de un marco de referencia total. Ochaita $(1980,1984)$ puso de manifiesto que a pesar de las numerosas investigaciones que existen sobre el tema, hay muy pocas que se dediquen con detenimiento y sistematización al estudio de la génesis de la representación del entorno, de los mapas cognitivos que los individuos carentes de 
visión tienen del espacio en que se mueven habitualmente. Aparte del estudio de Kephart et al. (1974), y de Dodds, Howart y Carter (1982) que ya reseñamos anteriormente, poco más sobre este tema podemos añadir, si acaso los resultados y conclusiones que reseñan Hollyfield y Foulke (1983). Según estos autores los ciegos tardan más años en poder aprender y recordar los mojones que delimitan una ruta nueva que los niños videntes.

\section{Influencia de la Experiencia Visual}

Warren, Annoshian y Bollinger (1973) reseñaron las diferencias que existían entre los experimentos de «espacio cercano» y los referidos al «espacio lejano», en el sentido de que los ciegos tardíos obtenían unos mejores resultados en las pruebas de espacio cercano que los ciegos de nacimiento, mientras que en las pruebas de espacio lejano estas diferencias no existían. Autores como Cleaves y Royal (1979) y Ochaita (1982) sugieren que ambas realizaciones no evalúan entonces los mismos problemas espaciales, las mismas habilidades. Las tareas complejas de movilidad en el espacio lejano dependerían menos de la experiencia visual que las del espacio abarcable con las manos. Se han encontrado resultados concordantes con estas suposiciones en los experimentos de Worchel (1951); Gomulicki (1961); Jourmaa (1965); Lockman, Rieser y Pick (1981); Warren y Kocon (1974); Drummond (1975); y Hollyfield y Foulke (1983).

Sin embargo, en otras investigaciones se han hallado pequeñas diferencias entre los ciegos congénitos y los tardíos: estos últimos ejecutaban mejor tareas de movilidad en rutas que los invidentes de nacimiento (Pick, 1974; Werner, 1974). Dichos autores afirmaban que tales diferencias indicaban que el mapa cognitivo de un lugar, esto es, la información acerca de la localización relativa de los lugares y las cosas, se representaba mejor en términos visuales. Dicho en otras palabras, según Hermelin y O'Connor (1982) el sistema sensorial implicado determina las propiedades organizacionales de codificación, representación y estructuración del entorno. Asî la estimulación sensorial táctil permite una organización relativa, subjetiva y egocéntrica del espacio, mientras que la estimulación o la experiencia visual proporciona una organización estable y abstracta del espacio. Rieser, Guth y Hill (1982) también encontraron diferencias en el mismo sentido entre ambos grupos de deficientes visuales en pruebas de actualización perceptiva. Los videntes y los ciegos tatdíos pueden recurrir a su aprendizaje perceptivo-visual para actualizar su posición conforme caminan, lo que les permite una mejor realización que los ciegos congénitos. Estos autores intentan establecer de forma solamente especulativa el rango de edad en la cual la experiencia visual que se tenga puede ser suficiente para una buena actualización perceptiva, y cifran en los ocho primeros años de la vida como el tiempo mínimo imprenscindible para tal actualización. En otro estudio de rutas Dodds, Howarth y Carter (1982), encontraron que a la hora externalizar el camino recorrido, los ciegos congénitos tendían a adoptar en su mayoría una estrategia más primitiva que la que usaban los ciegos tardíos, esto es, una estrategia de codificación egocéntrica ${ }^{2}$. Hay que destacar que en este experimento el número de sujetos fue muy reducido y que de cinco ciegos congénitos uno sí fue capaz de adoptar estategias parecidas a los invidentes del otro grupo.

De todas formas, en estos últimos estudios los ciegos de nacimiento fueron capaces de realizar con cierta corrección las tareas, lo que indica a nuestro parecer que la experiencia visual sólo facilita de alguna manera la movilidad, pero la habilidad o las estrategias cognitivas que se encuentran en la base de dicha mo- 
vilidad son funcionalmente parecidas para los dos grupos. Como afirma el propio Dodds: sla experiencia visual no es una condición necesaria para representarse un trazado espacial adecuadamente, pero ayuda a desarrollar una estrategia de codificación espacial externas. Quizá dentro de los trabajos de espacio lejano haya que diferenciar entre los que son estudios de movilidad en rutas y los que incluyen alguna forma de conocimiento espacial. Si se hace esta diferenciación, nos damos cuenta que en la mayoría de los trabajos de movilidad -cfr. Drummond (1975), Hollyfield y Foulke (1983) - no aparecen diferencias claras entre ciegos tardíos y ciegos de nacimiento y en cambio en trabajos de representación espacial estas diferencias son más usuales. Probablemente sea debido a que a la hora de externalizar un conocimiento espacial, el hecho de tener alguna experiencia visual facilite una manifestación de la representación más ajustada.

Por otro lado, en estos últimos experimentos no se trabajaba con videntes a los que se les privaba de la visión y este hecho puede llevar a relativizar más las conclusiones de tales estudios. Por último las diferentes metodologías utilizadas en todas estas investigaciones y la falta de un marco teórico general hacen que todos estos datos no se puedan considerar del todo concluyentes.

\section{CONCLUSIONES}

El intentar esbozar unas conclusiones coherentes y claras de los numerosos trabajos que aquí se han revisado resulta una tarea relativamente complicada. En ellos se mezcla de manera casi indisoluble problemas teóricos complejos que aún no están del todo resueltos en la Psicología cognitiva actual, con variables relativas a los sujetos (edad, experiencia visual, modalidad educativa, inteligencia, etc...) y con variables relativas al espacio (grande/pequeño, conocido/desconocido, cercano/lejano, etc....). Todo ello se complica a su vez con los distintos puntos de partida teóricos que toman los investigadores y con la heterogeneidad de los diseños que, a menudo, son difícilmente comparables entre sí. A continuación se van a tratar estos problemas de forma ordenada, en un intento de clarificar al lector las cuestiones más importantes que todavía hoy quedan sin aclarar y qué tipo de investigaciones deberían hacerse para intentar resolverlas. Se señalarán también las repercusiones educativas que de estos trabajos se derivan, así como aspectos relativos a la educación que, a nuestro juicio, necesitan ser estudiados.

Empezaremos por los problemas teóricos de carácter más general, para ir posteriormente tratando aspectos más concretos y puntuales.

Uno de los problemas más importantes que subyacen en los distintos trabajos revisados es el de las relaciones entre actividad espacial real o movilidad (para algunos autores «mapa conductual»), la representación mental que los sujetos tienen del entorno en que realizan tal actividad («mapa cognitivo»), y la externalización o exteriorización de tal representación mental. La complejidad de este programa a la hora de estudiar el conocimiento del entorno en las personas videntes ha sido señalado por numerosos autores (por ejemplo Marchesi, 1984 y de Vega, 1984). En el caso de las personas ciegas tal complejidad se multiplica a la vez que se hace necesario contar con ideas claras al respecto tanto a la hora de diseñar los experimentos como cuando se han de obtener conclusiones. Como ya hemos dicho la heterogeneidad en los resultados de los diversos autores puede deberse, en gran medida, a lo que estos entienden por representación, así como a los diferentes métodos de externalización que utilizan. 


\section{4}

Estos problemas se entrelazan con variables tales como la cantidad de la experiencia visual o la edad de los sujetos, dando lugar a resultados que no pueden ser concluyentes. Asi pues, resulta absolutamente imprescindible realizar un trabajo en el que se comparen las diversas formas de externalizar el conocimiento del entorno en orden a encontrar aquellas que puedan ser más idóneas en el caso de los ciegos. En cualquier caso es importante que los niños con problemas visuales graves utilicen al máximo las diversas formas de externalizar las representaciones tales como dibujo, construcciones, maquetas, etc.

Otra de las cuestiones teóricas en las que no existe acuerdo entre los distintos autores es si existe una modalidad de representación espacial diferente para cada una de las modalidades sensoriales (visual, auditiva, háptica y cinestésica), o si, por el contrario, es más conveniente hablar de una representación más general, amodal o multimodal, independientemente del sistema sensorial con que se tome la información y cuya naturaleza es de carácter espacial. Se trata éste de un tema importante, aunque de difícil resolución, ya que buena parte de las dificultades que tienen los invidentes en problemas de tipo espacial pueden derivarse de la necesidad de tomar la información con el tacto y de los problemas que origina la representación y el almacenamiento en la memoria de esa información sucesiva, lenta y fragmentaria.

En estrecha relación con este tema se encuentra el problema de las relaciones entre el denominado espacio cercano y el espacio lejano. De los estudios revisados en este artículo puede concluirse que las relaciones entre ambos espacios resultan especialmente complejas para los invidentes a causa de los distintos sistemas sensoriales implicados en la toma de información acerca del entorno cercano y en el entorno distante. En el caso de que existiera una representación amodal o multimodal, o de que ésta se desarrollase en una determinada etapa del desarrollo, sería conveniente fomentarla al máximo en las personas ciegas ya que las relaciones entre espacio cercano y lejano están relacionados con aspectos educativos sumamente importantes tales como, por ejemplo, la utilización de planos y representaciones cartográficas para orientar la movilidad de los invidentes, o el uso de sistemas cartográficos para el estudio de la geografía.

El fenómeno denominado «trasposición» (Jourmaa, 1973; Hermelin y O'Connor, 1982), está también vinculado a lo tratado anteriormente. Parece claro que la capacidad de las personas con cierta experiencia visual para traducir los datos obtenidos mediante el tacto o el movimiento a imágenes visuales, ha de estar en relación con el tipo de almacén espacial que se disponga. A pesar de su importancia y de su gran cantidad explicativa, la trasposición ha sido escasamente estudiada de forma experimental. Así pues se necesita nuevas investigaciones en orden a optimizar su utilización en la educación de los ciegos tardíos.

Por otra parte, sería también importante profundizar más en el estudio de los sistemas sensoriales que están implicados en la movilidad en ausencia de la visión, así como en el conocimiento de las representaciones espaciales que generan.

Con estos problemas de carácter más general o teórico se mezclan variables de sujeto que, por las características de la población de ciegos, resultan a menudo difíciles de comprobar de forma exacta, dando lugar a resultados experimentales que no siempre pueden considerarse concluyentes.

Una de estas variables es el tiempo de experiencia visual previo a la ceguera. Dado que una gran mayoría de las investigaciones dedicadas al estudio de la especialidad de los invidentes están orientadas a determinar si la experiencia 


\section{5}

visual es necesaria para elaborar representaciones espaciales de entorno cercanos y lejanos, se trata de un tema que debe controlarse al máximo en el diseño de las mismas. En términos generales podemos concluir que la experiencia visual facilita la representación espacial cuando ésta se evalúa con unos métodos de externalización cuya adecuación a los invidentes no está totalmente comprobada. Cuando se trata de movilidad y orientación en rutas nuevas, la experiencia visual no parece imprescindible. En cualquier caso, es necesario investigar en la utilización de técnicas de externalización no demasiado cargadas visualmente.

Otra de las variables que deben incidir en estas investigaciones es la edad $y$, sin embargo, en la mayoría de ellas no se ha tenido en cuenta. Desde nuestro punto de vista, el abordar el tema del conocimiento espacial en los ciegos desde una perspectiva genética puede clarificar en gran medida los aspectos teóricos del problema a que se ha aludido más arriba. Además, permitiría elaborar programas educativos más exactos tanto en lo referente al espacio cercano (rotación de formas, utilización del esquema corporal como sistema de referencia, etc.), en los programas de movilidad del entorno, así como el momento idóneo de establecer relaciones entre ambos utilizando maquetas, planos y mapas im. prescindibles para optimizar la movilidad en espacios desconocidos y para el estudio de materias tales como la geografía.

Además, existen otro tipo de variables individuales, como la inteligencia, la personalidad, las relaciones sociales, etc..., que como afirma Warren (1984), pueden explicar la gran variabilidad intrasujeto que a menudo se obtienen en las investigaciones con personas ciegas.

Para concluir, pasemos a revisar aquellas variables del entorno cuya incidencia en los trabajos sobre espacialidad de los invidentes no pueden considerarse definitivamente aclaradas. Dejando aparte las relaciones entre espacio cercano y lejano, uno de los aspectos peor estudiados es la incidencia del tamaño del entorno en la capacidad de representación de los invidentes. Mientras que en algunos trabajos se utilizan espacios relativamente pequeños, como una habitación o un laboratorio, en otros el ciego ha de moverse o representarse rutas de barrios o ciudades. Como se ha dicho anteriormente, es posible que los invidentes adultos sean capaces de conocer bien entornos relativamente pequeños $y$, sin embargo, tener problemas en coordinar esos pequeños espacios entre sí para elaborar la representación de un entorno más grande. Tales problemas estarían, además, en función de la edad y podrían ser objetos de sistemas de enseñanza, en orden a conseguir que los ciegos pudieran representarse el espacio con una estructura coordinada.

Por otra parte, si bien es posible que los ciegos se orienten en el espacio organizándolo mediante una estructura de rutas tal y como afirma Foulke (1982), sería necesario determinar si los invidentes utilizan para orientarse un tipo diferente al de los videntes, que pueden tener una utilidad similar.

Para terminar, sólo nos queda repetir la idea de que la utilización de instrumentos de ayudas y técnicas de entrenamiento para optimizar la movilidad de los invidentes han de estar en función de datos experimentales rigurosos que tengan en cuenta las distintas variables que inciden en el problema, en intima relación con una teoría que los justifique. 


\section{Notas}

1 Puede encontrarse un resumen de esta teoría en Ochaita (1983).

2 Para una mejor comprensión del desarrollo de las etapas del conocimiento del entorno en los niños (1.- Egocéntriça; 2.- Fija; 3.- Coordinada), véase por ejemplo Martín (1985), Evans (1980)..

\section{Referencias}

APPLEYARD, D. (1970). Styles and methods of structuring a city. Environment and Behavior, 2, $100-117$

BIRNS, S. L. (1986). Age and onset of blindness and development of space concepts: from topological to proyective space. Journal of Visual Impairment and Blindness, feb., 577-582.

BOWER, T. G. R. (1977). A primer of infant development. San Francisco: W.H. Freeman \& Co.

CARPENTER, P., y EISEMBERG, P. (1978). Mental totation and the frame of reference in blind and sighted individuals. Perception \& Psychopbysics, 23(2), 117-124.

CASEY, S. M. (1978). Cognitive mapping by the blind. Visual Impairment and Blindness, oct., 297-301.

Cleaves, W. T., y Royal, R. W. (1979). Spatial memory for configuration by congenitally blind, late blind, and sighted adults. Journal of Visual Impaiment and Blindness, 73, 13-19.

CoOlemAN, C. L., y WeINSTOCK, R. E. (1984). Physically handicapped blind people: adaptative mobility techniques. Joumal of visual Impairment and Blindness, mar., 113-117.

COOPER, L. A, y SHEPARD, R. N. (1975). Mental trasformations in the identification of left and right hands. Journal of Experimental Psycbology: Human Perception \& Performance, 1, 48-56.

DE VeGA. M. (1984). Introducción a la Psicología Cognitiva. Madrid: Alianza.

DodDS, A. G. (1983). Mental rotation and visual imagery. Joumal of visual Impairment and Blindnes, En., 16-18.

Dodds, A. G.; Howarth, C. I., y Carter, D. C. (1982). The mental maps of the blind: the role of previous visual experience. Joumal Of Visual Impairment and Blindness, En., 5-12.

DRUMOND, T. (1975). Visual and temporal strategies in blind children's apprehension of visual perspectives. Tesis doctoral no publicada. The Catholic University of America.

Evans, G. H. (1980). Environmental Cognition. Psychological Bulletin, 88(2), 259-287. (Trad. cast. en Estudios de Psicología, 1983, 14-15, 47-84).

Evans, G. W.; MARrero, D., y BUTLER, P. (1981). Environment learning and cognitive mapping. Environment and Behavior, 13(1), 83-104.

FARMER, L. (1980). Mobility devices. En R. Welsh y B. Blasch (Eds.): Foundation of Orientation and Mobility (pp. 357-412). Nueva York. American Foundation for the Blind.

FLETCHER, J. F. (1981). Spatial representation in blind children: Effects of tasks variations. Joumal of Visual Impairment and Blindness, $75,1-3$.

FOULKE, E. (1971). The perceptual basis for mobility. American Foundation for the Blind. Research Boulletin, $23,1-8$.

FoulKe, E. (1982). Perception, cognition and mobility of blind pedestrian. En M. Potegal (Ed.): Spatial abilities. Nueva York: Academic Press.

GoumulCKI, B. R. (1961). The development of perception and learning in blind children. The Psychological Laboratory, Cambridge University.

HART, R. (1979). Children's Experience of Place. Nueva York: Irvington.

Herman, J. E.; Herman, T. G., y Chatman, M. A. (1983). Constructing cognitive maps from partial information: A demostration study with congenitally blind subjects. Joumal of Visual Impairment and Blindness, May., $195-198$.

HERMELIN, B., y O'CONNOR, N. (1975). Localization and distance estimates by blind and sighted children. International Joumal of Experimental Psychology, 27, 295-301.

Hermelin, B., y O'CONNOR, N. (1982). Spatial modality coding in children with and without impairments. En M. Potegal (Ed.) Spatial Abilities. Nueva York: Academic Press.

HOLlYFIELD, R. L., y FoULKE. E. (1983). The spatial cognition of blind pedestrians. Journal of Visual Impairment and Blindness, may., 204-210.

JOURMAA. J. (1965). An analysis of the components of orientation and mental manipulation of spatial relationships. Rep. Institute of Occupational Health, Hensilki, 28.

JourmaA. J. (1973). Trafisposition in mental spatial manipulation. A theoretical analysis. American Foundation for the Blind. Research Boulletin, 26, 87-134.

JoURMA . J., y SUONIO, K. (1969). Optification tendency in tactual spatial manipulation: An experimental study. Rep. Institute of Occupational Health, Helsinki, 69.

Kephart, J. G.; Kephart, C. P., y Scharz, G. C. (1974). A journey into the wold of the blind Child. Exceptional Children. Marzo, 421-428.

MARCHESI, A. (1983). Conceptos espaciales, mapas cognitivos y orientación en el espacio. Estudios de Psicología, 14/15, 85.92. 
MARMOR. G. S., y ZABACK. L. A. (1976). Mental rotation by the blind: Does mental representation depend on visual imagery? Joumal of Experimental Psychology: Human Perception and Performance, 2, (4), 515-521.

MARTIN, E. (1985). La representación espacial del entorno en los niños. Una comparación entre el medio urbano y el medio rural. Tesis Doctoral inédita. Universidad Complutense.

MeITZAR. J., y SHEPARD, R. N. (1974). Transformational studies of the internal representations of three-dimensional objects. En R.L. Solso (ed.): Theories in cognitive psychology (The Loyola Symposium) Potomac, Md.: Erlbaum.

MiLiAR. S. (1976). Spatial representation by blind and sighted children. Joumal of Experimental Child Psychology, 21, 460-479.

MILIAR, S. (1979). The utilization of external and movement cues in simple spatial tasks by blind and sighted children. Perception, 8, 11-20.

MiLAR, S. (1982). The problem of imagery and spatial development in the blind, De Gelder (Ed.): Representation And Cognition.

O'Connor. N., y Hermelin. B. M. (1975). Modality specific spatial coordinates. Perception \& Psychopbysics, 17(2), 213-216.

Ochaita, E. (1982). El conocimiento del espacio en los niños ciegos. Tesis Doctoral inédita. Universidad Autónoma de Madrid.

Ochaita, E. (1983). La teoría de Piaget sobre el desarrollo del conocimiento espacial. Estudios de Psicología, 14/15, 93-108.

OCHAItA, E. (1984). Una aplicación de la teoría piagetiana al estudio del conocimiento espacial en los niños ciegos. Infancia y Aprendizaje, 25, 80-92.

OChalta, E. (1986). Conocimiento del espacio y enseñanza de la geografía. Ponencia presentada a las II Jomadas Intemacionales de Psicología y Educaciön. Madrid.

PICK, A. D., y PICK, H. L. (1966). A developmental study of tactual discrimination in blind and sighted children and adults. Psychonomic Science, 6, 367-368.

PICK, A. D.; KLEIN, R. E., y PICK, H. L. (1968). Visual and tactual identification of form orientation. Joumal of Experimental Child Psychology, 4, 391-397.

RIESER. J. J.; LOCKMAN, J. J., y PICK. H. L. (1981). The role of visual experience in knowledge of spatial layout. Perception \& Psychopbysics, 28, 185-190.

RIESER, J. J.; GUTH, D. A., y HILL, E. W. (1982). Mental processes mediating independent travel: implications for orientation and mobility. Joumal of visual Impaiment and Blindness, jun, 213-218.

Revesz, G. (1950). Psychology and Art of the Blind. Toronto: Logmans.

ROSENCRANZ, D., y SuSLICK, R. (1976). Cognitive models for spatial representations in congenitally blind, adventitiously blind, and sighted subjets. New Outlook for the Blind, 70, 188-194.

ShaGEN, (1970). Kinesthetic Memory, Comparing. Blind and Sighted Subjects. Tesis Doctoral No Publicada. George Washington University.

SIEGEL. A. W., y WHITE, S. H. (1975). The development of spatial representation of large-scale enviorements. En H.W. Reese (ed), Advances in child development and behavior. (vol 10), N. York, Academic Press.

SIMPKINS, K. E., y SIEGEI, A. J. (1979). The blind child's construction of ten proyective straight line. Joumal of Visual Impaiment and Blindness, 73, 233-238.

SuTERKo (1973). Life Adjustment. En B. Lowenfeld (Ed.) Visually Handicapped Child In School. Nueva York: John Day Co.

VON SENDFN, M. (1960). Space and sight: The perception of space and shape in the congenitally blind before and after operation. Glencoe, IL: The Free Press, 1960.

W ARREN, D. H. (1978). Perception of the blind. En E. Carterette y T. Friedman (Ed): Handbook of Perception. Vol. X Perceptual Ecology. Nueva York: Academic Press.

W ARREN, D. H. (1984). Blindness and early childhood development. Nueva York: American Foundation for the Blind.

Warren, D. H.; ANOOShian, L. M., y Bounger, J. G. (1973). Early vs. late blindness. The role of early vision in spatial behavior. AFB Research Boulletin, 26, 151-170.

W ARREN, D. H., y KOCON. J. A. (1974). Factors in the successful mobility for the blind: a review. AFB. Research Boulletin, 28, 191-218.

WEINSTOCK. R. (1982). Resources on mobility for the physically handicapped. Joumal of Visual $\mathrm{Im}$ pairment and Blindness, 76, 317-318.

WORCHEL, P. (1951). Space perception and orientation in the blind. Psychol. Monogr. 65, 15.

\section{Extended summary}

In this paper we have attempted to offer the reader a critical review of the most important pieces of work related to space and blindness. The first part concerns research on near space, that is to say, on the space that the visually impaired person can reach with his/her hands. In form-rotation tasks the different research projects demostrate that while the visual experience aids the un- 
derstanding of this type of task, it is not essential, since the congenitally blind manage to do these tasks in a satisfactory manner. With reference to research on the development of spatial knowledge from a piagetian perspective, it has to be pointed out that the visually impaired person at adolesence is able to understand topological and metrical spatial relationships perfectly.

In the section on far space we have analysed the different studies in which the blind have to deal with spatial relationships in a more or less extensive environment - that which is not within arm-reach, and they have to place themselves within this environment from a static position on the basis of cues given by movement, propioception and hearing. Research on orientation and mobility, space representation and cognitive mapping is reviewed, as well as the role of the visual experience in the understanding of spatial relationships in extensive environments.

Concerning mobility, the spatial conduct in the strict sense requires the functioning of very different processes: perceptive, motor, associative and mnesic processes. Given that this is a particularly complex process without vision and one in which the blind person tends to have great difficulty, explanatory hypotheses are offered. Technical aids designed to help orientation and mobility such as special walking-sticks and electronic aids are also reviewed.

With respect to the representation of the environment, the research work concerning the nature of the representations of the immediate environment of the visually impaired is discussed. In general terms, it can be said that the blind, even those blind from birth, are able to elaborate cognitive maps or topographical representations of the spaces in which the move. The most important variables involved in the environmental representation capacity - those relating to the subject- age, visual experience, space experience, and also those relating to the environment size, complexity, are analysed. Special attention is given to the visual experience variable. The various techniques of externalization of spatial representation which are susceptible to use with the blind are examined.

The final part of the paper outlines some conclusions where the aim is to specify what the most important theoretical and methodological problems presented by research on spatial knowledge in the blind are due to. Theoretical problems concern the relationship between mobility and representation and the modal or intermodal character of spatial representation and the relationship between near and far space in the visually impaired and the phenomenom of «transpositions. Concerning methodological problems the importance of subject and environmental variables are analysed with reference to the results of the studies reviewed. 